\title{
ANÁLISIS DE EFECTOS SÍSMICOS ORTOGONALES HORIZONTALES EN TERRENO BLANDO
}

\author{
Jesús Valdés González ${ }^{(1)}$ y Mario Ordaz Schroeder ${ }^{(2)}$
}

\begin{abstract}
RESUMEN
En este artículo se estudia el problema que representa la combinación de los efectos sísmicos en las estructuras causados por la acción simultánea de los dos componentes ortogonales horizontales del movimiento del suelo. Mediante el uso de la teoría de vibraciones aleatorias se desarrollan algunas expresiones analíticas que permiten combinar los efectos sísmicos causados por la acción de ambos componentes ortogonales horizontales del movimiento del suelo con el propósito de estimar la máxima respuesta elástica, bi-direccional de la estructura. La principal hipótesis que se hace en el desarrollo de estas expresiones, se refiere a la representación del espectro de amplitudes de Fourier de ambos componentes del movimiento del suelo mediante funciones tipo delta de Dirac. Por medio del análisis elástico bi-direccional, paso a paso de distintos modelos estructurales, se verifica la precisión del planteamiento de combinación propuesto. El procedimiento desarrollado es aplicable exclusivamente al caso de suelo blando y considera explícitamente el ángulo de incidencia del temblor y el tipo de respuesta que se analiza (ortogonal o colineal).
\end{abstract}

\begin{abstract}
This work studies the problem that represents the combination of the seismic effects on structures caused by the action of two horizontal orthogonal components of the ground motion. By using random vibrations some analytical expressions were developed to combine the two horizontal orthogonal seismic effects in order to estimate the elastic bi-directional peak response. The main hypothesis made in the development of these expressions was to represent the Fourier's spectrum for the two orthogonal components of the ground motion by a Dirac's delta. Through the fully bidirectional elastic "step by step" analysis of some structural models, it is verified the accuracy of the proposed method. The exposed procedure is exclusively applicable to soft soil. It considers explicitly the angle of earthquake incidence and the kind of response in terms of the direction of its components (orthogonal or collinear).
\end{abstract}

\section{INTRODUCCIÓN}

Durante la ocurrencia de un temblor, las estructuras se ven sometidas a un campo complejo del movimiento del suelo. Sin embargo, en la práctica es común analizarlas, únicamente, bajo la acción de dos componentes ortogonales horizontales del movimiento del suelo. En algunos casos (regiones cerca del epicentro del temblor, por ejemplo) se incluye un tercer componente ortogonal, el cual actúa en la dirección vertical.

Artículo recibido el 14 de noviembre de 2007 y aprobado para su publicación el 20 de noviembre de 2008. Se aceptarán comentarios y/o discusiones hasta cinco meses después de su publicación

(1) Facultad de Ingeniería, Universidad Autónoma del Estado de México, Cerro de Coatepec S/N, Ciudad Universitaria, Toluca, México, CP. 50130,jvaldes@uaemex.mx.

${ }^{(2)}$ Instituto de Ingeniería, Universidad Nacional Autónoma de México, México, D.F, 04510, mors@pumas.iingen.unam.mx 
Cuando el análisis de la estructura se lleva a cabo mediante técnicas espectrales, la mayoría de los códigos recomiendan analizar la estructura en forma independiente en dos direcciones ortogonales para obtener las máximas respuestas unidireccionales que interesen (máximas respuestas unidireccionales). Bajo este criterio, el principal problema es cómo combinar las máximas respuestas unidireccionales $\left(R_{x}\right.$ max y $R_{y} \max$ en la figura 1) para estimar la máxima respuesta bi-direccional ( $R_{x y \max }$ en la figura 1$)$.
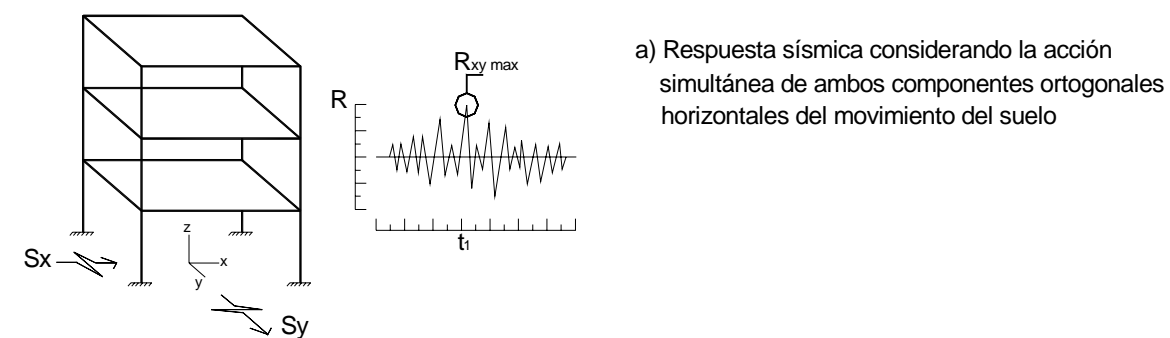

a) Respuesta sísmica considerando la acción simultánea de ambos componentes ortogonales horizontales del movimiento del suelo
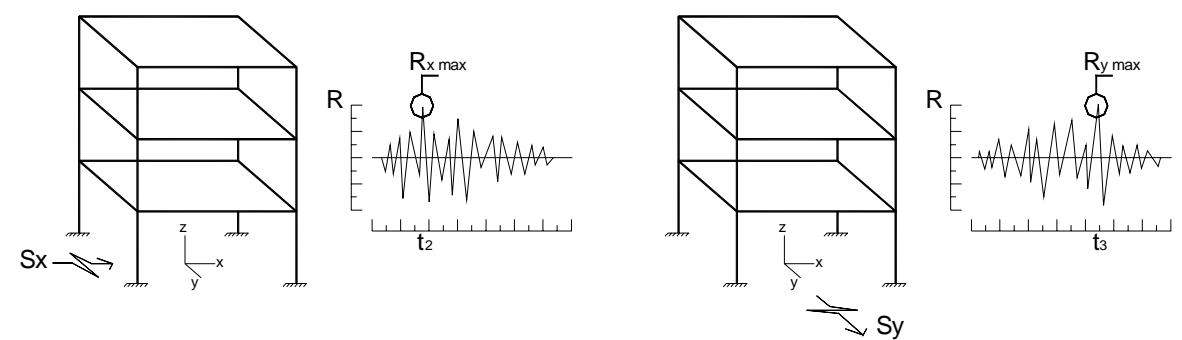

b) Respuesta sísmica considerando únicamente la acción del movimiento del suelo en la dirección $\mathrm{x}$

c) Respuesta sísmica considerando únicamente la acción del movimiento del suelo en la dirección y

Figura 1. Respuesta sísmica unidireccional y bi-direccional

Los códigos de diseño especifican diferentes procedimientos para estimar la máxima respuesta bidireccional a partir de la combinación de las máximas respuestas unidireccionales calculadas mediante métodos espectrales. Los de mayor uso son las reglas de combinación de porcentajes a. En estos métodos, la máxima respuesta bi-direccional se estima combinando los efectos del $100 \%$ de la máxima respuesta causada por la acción del sismo en una dirección (respuesta unidireccional), y $\alpha$ veces la máxima respuesta causada por la acción del sismo en la dirección ortogonal.

Rosenblueth y Contreras (1977) propusieron un valor de $\alpha=30 \%$, valor que ha sido adoptado por distintos códigos de diseño (NEHRP, 1997; COVENIN, 1998; Caltrans, 1990; RDF, 1993). Por su parte, Newmark (1975) propuso un valor de $\alpha=40 \%$, el cual fue adoptado por otros códigos o especificaciones de diseño (ASCE, 1986; Nutt, 1996). Adicionalmente, algunos códigos especifican una tercer regla, la cual estima la máxima respuesta bi-direccional como la raíz cuadrada de la suma de las máximas respuestas unidireccionales elevadas al cuadrado (SRSS) (ASCE, 1986; ICBO, 1997).

Con base en el trabajo de Smeby y Der Kiureghian (1985), Menun y Der Kiureghian (1998) propusieron una regla de combinación modal para sistemas elásticos (regla CQC3), la cual toma en cuenta explícitamente la correlación entre las respuestas modales y la correlación entre los componentes horizontales del movimiento del suelo. La ecuación que proporciona esta regla para estimar la respuesta es función del ángulo de incidencia del temblor. López y Torrres (1997) desarrollaron una ecuación que permite obtener el ángulo de incidencia que produce la máxima respuesta, al utilizar esta regla. 
López, Chopra y Hernández (2000) desarrollaron una fórmula para calcular la respuesta crítica, la cual no requiere de la evaluación explícita del ángulo crítico en la regla original CQC3. Esta fórmula depende de la relación entre las formas de los espectros, de la relación entre los componentes de la respuesta y de la correlación entre los componentes de la respuesta causados por la acción de los componentes ortogonales del movimiento del suelo. Para los propósitos de este trabajo, esta fórmula simplificada será referida como regla CQC3.

Ninguna de las reglas de combinación mencionadas ( $\alpha$, SRSS y CQC3) considera el tipo de respuesta que se analiza en relación a la dirección de sus componentes (colineal u ortogonal), ni las condiciones del tipo de suelo. Algunos estudios (Heredia-Zavoni y Machicao-Barrionuevo, 2004; Valdés, 1999) han demostrado la relevancia de estas variables en la respuesta sísmica de las estructuras. HerediaZavoni y Machicao-Barrionuevo (2004) analizaron el efecto de los componentes ortogonales horizontales del movimiento del suelo en la respuesta elástica de sistemas con torsión, rígidos y flexibles, en dos tipos de suelo (duro y blando). En este estudio se demostró que la respuesta bi-direccional era diferente en función de las propiedades dinámicas del sistema, así como de las condiciones del suelo. También examinaron las reglas de combinación de porcentajes y encontraron que en algunos casos dichas reglas pueden sobrestimar las fuerzas de diseño y en otros subestimarlas.

Por su parte, Reyes-Salazar et al. (2004) evaluaron las reglas SRSS y la de combinación de porcentajes $(\alpha=30 \%)$ a partir del análisis elástico e inelástico de cuatro marcos tridimensionales de acero resistentes a momento, sometidos a la acción simultánea de ambos componentes ortogonales horizontales de distintos registros sísmicos. Analizaron diferentes tipos de respuesta como la fuerza cortante basal (ortogonal) y la carga axial en columnas interiores, exteriores y de esquina (colineal). Los resultados mostraron que ambas reglas pueden subestimar el efecto combinado de ambos componentes ortogonales horizontales del movimiento del suelo. Las mayores subestimaciones se presentaron al utilizar la regla SRSS y fueron más importantes al analizar la respuesta inelástica de las estructuras. Concluyen que se deben modificar los requerimientos de diseño sísmico para estimar la respuesta combinada, que se basen en dichas reglas. Proponen utilizar una regla de combinación de porcentajes con $\alpha=40 \%$.

La importancia de diferenciar el tipo de respuesta (colineal u ortogonal) en la combinación de efectos sísmicos ortogonales ha sido estudiada y reconocida en diferentes trabajos (Reed y Kennedy, 1996; Valdés, 2004).

Otra variable que influye en la respuesta estructural, es la orientación de los ejes a lo largo de los cuales se consideran actuando ambos componentes ortogonales horizontales del movimiento del suelo. Wilson et al. (1995) demostraron que las fuerzas de diseño que resultan al utilizar las reglas de combinación de porcentajes ( $\alpha=30 \%$ o $40 \%$ ), dependen de la orientación de dichos ejes. Sin que las propias reglas proporcionen una recomendación para elegir esta orientación. A su vez, demostraron que la regla SRSS proporciona fuerzas de diseño que no dependen de la orientación de los ejes de la estructura, por lo que recomiendan utilizar dicha regla en lugar de las de combinación de porcentajes. Esta otra variable, al igual que los tipos de terreno y respuesta se consideran explícitamente en el procedimiento que a continuación se presenta.

\section{TIPOS DE RESPUESTA (ORTOGONALES O COLINEALES)}

De acuerdo a la dirección de sus componentes, las distintas respuestas estructurales se pueden clasificar en dos tipos; ortogonales o colineales. Las respuestas ortogonales corresponden a aquellos casos donde los dos componentes de la respuesta actúan de manera ortogonal, uno respecto al otro (figura 2a). 
El término componente se refiere a la respuesta estructural (fuerza, esfuerzo o deformación) generada por la acción unidireccional del movimiento del suelo asociado a uno de los dos ejes ortogonales de la estructura, $x$ o $y\left(R_{x}\right.$ y $R_{y}$ respectivamente).

Las respuestas colineales corresponden a aquellos casos donde ambos componentes de la respuesta estructural actúan en la misma dirección (figura $2 b$ ). La forma en que se calcula la respuesta bi-direccional $\left(R_{x y}\right)$ de la estructura ocasionada por la acción simultánea de ambos componentes $\left(R_{x} \mathrm{y} R_{y}\right)$ es diferente en cada caso.

Algunos ejemplos de respuestas de tipo ortogonal son: la fuerza cortante basal, el desplazamiento absoluto de un nodo, la fuerza cortante que actúa en columnas circulares o tornillos, etc. Como ejemplos de respuesta colineal están los siguientes: la fuerza axial en columnas, el momento flexionante alrededor de un mismo eje en vigas y columnas, el momento de torsión en vigas y columnas, la fuerza axial en arriostramientos, etc.

La respuesta bi-direccional considerando la acción simultánea de ambos componentes ortogonales horizontales del movimiento del suelo en cualquier instante $t, R_{x y}(t)$, se obtiene mediante las siguientes expresiones. Cuando ambos componentes de la respuesta estructural son ortogonales (figura 2a):

$$
R_{x y}(t)=\sqrt{R_{x}^{2}(t)+R_{y}^{2}(t)}
$$

Cuando los componentes de la respuesta estructural son colineales (figura 2b):

$$
R_{x y}(t)=R_{x}(t)+R_{y}(t)
$$

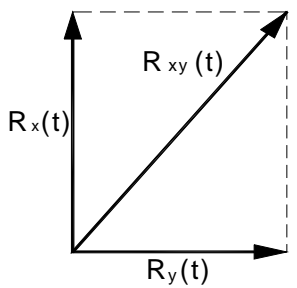

a) Respuesta ortogonal

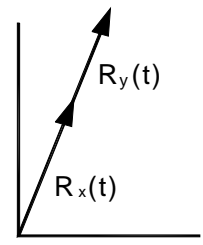

b) Respuesta colineal

Figura 2. Tipos de respuesta; $t$ denota al tiempo

\section{FORMULACIÓN DEL PROBLEMA}

Se estudian dos variables. Una de estas variables es $\gamma$, la cual se define como el cociente de la máxima respuesta bi-direccional considerando la acción simultánea de ambos componentes ortogonales horizontales del movimiento del suelo $\left(R_{x y}(t)_{\max }\right)$ entre la máxima respuesta unidireccional considerando la acción de un solo componente ortogonal horizontal del movimiento del suelo $\left(R_{x}(t)_{\max }\right.$ o $\left.R_{y}(t)_{\max }\right)$ (Valdés, 1999).

$$
\gamma=\operatorname{Min}\left\{\frac{R_{x y}(t)_{\max }}{R_{x}(t)_{\max }}, \frac{R_{x y}(t)_{\max }}{R_{y}(t)_{\max }}\right\}
$$


La otra variable es $\alpha$, la cual se calcula de la siguiente forma

$$
\alpha=\operatorname{Min}\left\{\frac{R_{x y}(t)_{\text {max }}-R_{x}(t)_{\text {max }}}{R_{y}(t)_{\max }}, \frac{R_{x y}(t)_{\max }-R_{y}(t)_{\max }}{R_{x}(t)_{\max }}\right\}
$$

Min\{.\} denota la mínima de las cantidades indicadas.

\section{VIBRACIONES ALEATORIAS}

En el dominio de la frecuencia, cualquier respuesta de interés de una estructura sometida a la acción simultánea de ambos componentes ortogonales horizontales del movimiento del suelo puede calcularse de la siguiente forma: Si los componentes de la respuesta son colineales, entonces

$$
R_{x y}(\omega)=g_{1}(\omega) x_{s}(\omega)+g_{2}(\omega) y_{s}(\omega)
$$

Si los componentes son ortogonales

$$
R_{x y}(\omega)=\sqrt{g_{1}^{2}(\omega) x_{s}^{2}(\omega)+g_{2}^{2}(\omega) y_{s}^{2}(\omega)}
$$

donde $g_{1}(\omega)$ y $g_{2}(\omega)$ son funciones deterministas que dependen de las características de la estructura (rigidez, amortiguamiento, geometría, distribución de masa, etc.), así como de la respuesta que en particular se calcule. $\ddot{x}_{s}(\omega)$ y $\ddot{y}_{s}(\omega)$ son las transformadas de Fourier de los componentes ortogonales horizontales de la aceleración del suelo.

En general, $R_{x y}(\omega)$ es un número complejo cuya amplitud al cuadrado está dada por:

$$
\left|R_{x y}(\omega)\right|^{2}=R_{x y}(\omega) R_{x y}^{*}(\omega)
$$

donde el símbolo * denota al conjugado de un número complejo.

Reemplazando las ecs. 5 o 6 en la ec. 7, resulta que, para el caso de componentes colineales

$$
\left|R_{x y}(\omega)\right|^{2}=g_{1}^{2}(\omega)\left|x_{s}(\omega)\right|^{2}+g_{2}^{2}(\omega)\left|y_{s}(\omega)\right|^{2}+2 g_{1}(\omega) g_{2}(\omega)\left|x_{s}(\omega)\right|\left|y_{s}(\omega)\right| \operatorname{real}[\phi(\omega)]
$$

y para componentes ortogonales:

$$
\left|R_{x y}(\omega)\right|^{4}=g_{1}^{4}(\omega)\left|x_{s}(\omega)^{4}+g_{2}^{4}(\omega)\right| y_{s}(\omega)^{4}+2 g_{1}^{2}(\omega) g_{2}^{2}(\omega)\left|x_{s}(\omega)\right|^{2}\left|y_{s}(\omega)\right|^{2} \operatorname{real}^{2}[\phi(\omega)]
$$

donde $\varphi(\omega)$ es la función de coherencia entre los componentes ortogonales horizontales de la aceleración del suelo. En general, los valores de la amplitud de la coherencia suavizada son bajos. Se ubican en un intervalo que va de 0.4 a 0.6, aproximadamente (Santa-Cruz et al., 1999a, b; Valdés, 1999 y 2003). 
Debido a que el interés de este trabajo se centra en analizar el movimiento del suelo en terreno blando, se asume que el espectro de amplitudes de Fourier de ambos componentes ortogonales horizontales del movimiento del suelo se puede aproximar mediante una delta de Dirac localizada en la frecuencia predominante del suelo. En la figura 3 se muestra el espectro de amplitudes de Fourier del movimiento del suelo registrado durante el sismo de 1985 en la estación SCT de la ciudad de México (terreno blando). La forma que tienen estos espectros justifica su aproximación mediante deltas de Dirac. Esta hipótesis implica que los dos componentes horizontales del movimiento del suelo sean caracterizados mediante señales cuyos espectros de Fourier tienen la misma forma (deltas de Dirac), pero cuyas amplitudes no son necesariamente las mismas. Es decir, se asume que

$$
k=\frac{\left|y_{s}(\omega)\right|}{\left|x_{s}(\omega)\right|}
$$

donde $k$ varía de 0.4 a 1.0 aproximadamente y depende de la orientación de los ejes que se utilicen para descomponer el movimiento del suelo (Valdés, 2004).

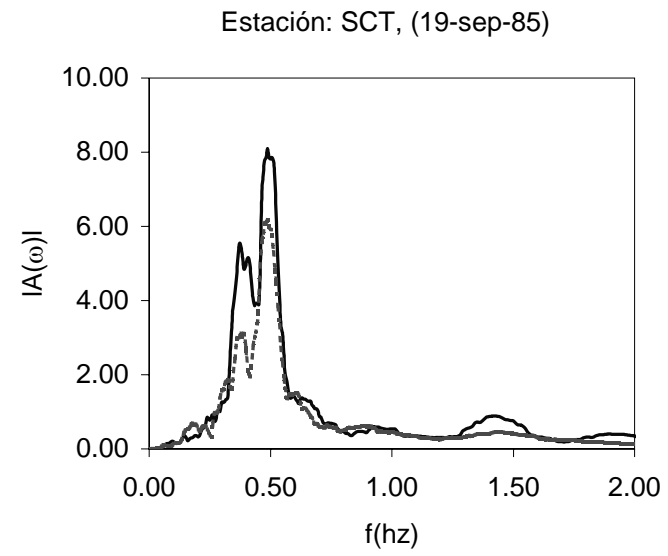

Figura 3. Espectro de amplitudes de Fourier correspondiente al registro obtenido durante el temblor del 19 de septiembre de 1985 en la estación SCT de terreno blando en la ciudad de México

Bajo estas consideraciones, las ecs. 8 y 9 pueden rescribirse de la siguiente forma. Para el caso de componentes colineales (ec. 8):

$$
\left|R_{x y}\left(\omega_{s}\right)\right|^{2}=g_{1}^{2}\left(\omega_{s}\right)+k^{2} g_{2}^{2}\left(\omega_{s}\right)+2 k g_{1}\left(\omega_{s}\right) g_{2}\left(\omega_{s}\right) \operatorname{real}\left[\phi\left(\omega_{s}\right)\right]
$$

Para componentes ortogonales (ec. 9):

$$
\left|R_{x y}\left(\omega_{s}\right)\right|^{4}=g_{1}^{4}\left(\omega_{s}\right)+k^{4} g_{2}^{4}\left(\omega_{s}\right)+2 k^{2} g_{1}^{2}\left(\omega_{s}\right) g_{2}^{2}\left(\omega_{s}\right) r e a l^{2}\left[\phi\left(\omega_{s}\right)\right]
$$

De acuerdo a la teoría de vibraciones aleatorias (Vanmarcke, 1976), el valor esperado de la máxima respuesta cuadrática considerando la acción simultánea de ambos componentes ortogonales horizontales del movimiento del suelo puede calcularse con la siguiente expresión: 


$$
E\left(R_{x y \text { max }}^{2}\right)=\frac{F_{p}}{2 \pi T_{s}} \int_{-\infty}^{\infty}\left|R_{x y}(\omega)\right|^{2} d \omega
$$

donde $F_{p}$ es el factor pico y $T_{s}$ la duración de la fase intensa del movimiento del suelo.

Evaluando la ec. 13 para el caso donde los componentes de la respuesta son colineales (ec. 11) resulta:

$$
E\left(R_{x y \max }^{2}\right)=\frac{F_{p}\left(g_{1}^{2}\left(\omega_{s}\right)+k^{2} g_{2}^{2}\left(\omega_{s}\right)+2 k g_{1}\left(\omega_{s}\right) g_{2}\left(\omega_{s}\right) \operatorname{real}\left[\phi\left(\omega_{s}\right)\right]\right)}{2 \pi T_{s}}
$$

Para el caso de componentes ortogonales (ec. 12):

$$
E\left(R_{x y \max }^{4}\right)=\frac{F_{p}\left(g_{1}^{4}\left(\omega_{s}\right)+k^{4} g_{2}^{4}\left(\omega_{s}\right)+2 k^{2} g_{1}^{2}\left(\omega_{s}\right) g_{2}^{2}\left(\omega_{s}\right) \operatorname{real}^{2}\left[\phi\left(\omega_{s}\right)\right]\right)}{2 \pi T_{s}}
$$

Nótese que la integral de la ec. 13 ha desaparecido de las ecs. 14 y 15 debido a que los espectros de amplitudes de Fourier del movimiento del suelo son deltas de Dirac.

Sí sólo actúa un componente del movimiento del suelo, entonces la respuesta puede calcularse como sigue. Para la acción del temblor en la dirección $x$ :

$R_{\chi}(\omega)=g_{1}(\omega) x_{s}(\omega)$

para la acción del sismo en la dirección $y$,

$$
R_{y}(\omega)=g_{2}(\omega) k y_{s}(\omega)
$$

El valor esperado de la respuesta cuadrática pico, considerando la acción individual de un componente del movimiento del suelo se puede calcular de acuerdo a las siguientes expresiones:

$$
\begin{aligned}
& E\left(R_{x \max }^{2}\right)=\frac{F_{p x} g_{1}^{2}\left(\omega_{s}\right)}{2 \pi T_{s x}} \\
& E\left(R_{y \text { max }}^{2}\right)=\frac{F_{p y} k^{2} g_{2}^{2}\left(\omega_{s}\right)}{2 \pi T_{s y}}
\end{aligned}
$$

donde $T_{s x}$ y $T_{s y}$ son las duraciones de las fases intensas del movimiento del suelo en cada dirección. Bajo estas condiciones, el cociente $\gamma$ (ec. 3) puede calcularse como sigue:

Si los componentes de la respuesta son colineales 


$$
\begin{aligned}
& \gamma_{c}=\frac{F_{1} \sqrt{\left(R_{x y \max }^{2}\right)}}{F_{2} \sqrt{E\left(R_{x \max }^{2}\right)}=\frac{E\left(R_{x y \max }\right)}{E\left(R_{x \max }\right)}} \\
& \gamma_{c}=\frac{F_{1}}{F_{2}} \sqrt{\frac{F_{p}\left(g_{1}^{2}\left(\omega_{s}\right)+k^{2} g_{2}^{2}\left(\omega_{s}\right)+2 k g_{1}\left(\omega_{s}\right) g_{2}\left(\omega_{s}\right) \operatorname{rea}\left[\phi\left(\omega_{s}\right)\right]\right)}{2 \pi T_{s}}} \\
& \frac{F_{p x} g_{1}^{2}\left(\omega_{s}\right)}{2 \pi T_{s x}}
\end{aligned}
$$

Si los componentes de la respuesta son ortogonales, entonces:

$$
\begin{aligned}
& \gamma_{o}=\frac{F_{1} \sqrt[4]{E\left(R_{x y \max }^{4}\right)}}{F_{2} \sqrt[4]{E\left(R_{x \max }^{4}\right)}=\frac{E\left(R_{x y \max }\right)}{E\left(R_{x \max }\right)}} \\
& \gamma_{o}=\frac{F_{1}}{F_{2}} \sqrt[\frac{F_{p}\left(g_{1}^{4}\left(\omega_{s}\right)+k^{4} g_{2}^{4}\left(\omega_{s}\right)+2 k^{2} g_{1}^{2}\left(\omega_{s}\right) g_{2}^{2}\left(\omega_{s}\right) r e a l^{2}\left[\phi\left(\omega_{s}\right)\right]\right)}{2 \pi T_{s}}]{\frac{F_{p x} g_{1}^{4}\left(\omega_{s}\right)}{2 \pi T_{s x}}}
\end{aligned}
$$

Las ecs. 20 y 21 fueron formuladas considerando que la respuesta individual debida a la acción del sismo en $x$ es mayor que la correspondiente a $y\left(R_{x \text { máx }}>R_{y \text { máx }}\right)$.

$F_{1}$ y $F_{2}$ son funciones que dependen de las distribuciones de probabilidad de las variables aleatorias que se estudian. El propósito de estas funciones es transformar la raíz cuadrada de los valores esperados de las máximas respuestas cuadráticas al valor esperado de la máxima respuesta. Para simplificar las ecs. 20 y 21 , se asume que $F_{1} / F_{2}=1$. Además, se considera que los factores pico $\left(F_{p}, F_{p x}\right)$ y las duraciones de las fases intensas $\left(T_{s}, T_{s x}\right)$ son los mismos, tanto para los efectos bi-direccionales como para los unidireccionales. De esta forma, la variable $\gamma$ puede calcularse de acuerdo a las siguientes expresiones. Si los componentes de la respuesta son colineales

$$
\gamma_{c}= \pm \sqrt{1+\beta^{2}+2 \beta \operatorname{real}\left[\phi\left(\omega_{s}\right)\right]}
$$

Si los componentes de la respuesta son ortogonales

$$
\gamma_{o}= \pm \sqrt[4]{1+\beta^{4}+2 \beta^{2} \operatorname{real}^{2}\left[\phi\left(\omega_{s}\right)\right]}
$$

donde

$$
\beta=\frac{k g_{2}\left(\omega_{s}\right)}{g_{1}\left(\omega_{s}\right)}
$$


Estas expresiones consideran que la respuesta unidireccional máxima ocurre para la acción del sismo en la dirección $x$. Por ello, $\gamma$ deberá multiplicarse por la máxima respuesta unidireccional relacionada a esta dirección, con el propósito de estimar la máxima respuesta considerando la acción bidireccional del sismo.

El signo \pm que aparece en las raíces de las ecs. 22 y 23 toma en consideración la posible ocurrencia del temblor en uno u otro sentido. La máxima respuesta bi-direccional deberá calcularse considerando ambas raíces de $\gamma$.

Es indispensable considerar la acción de ambos componentes ortogonales horizontales del movimiento del suelo actuando en ambos sentidos (+ o -). Por ello, deberán analizarse las cuatro combinaciones de signo que resultan al combinar ambos componentes ortogonales del movimiento del suelo. Esto representa dos posibilidades de signo para $\beta(+0-)$.

Cuando los componentes de la respuesta son colineales, el valor de $\gamma$ que resulta al considerar $\beta$ con signo positivo no es el mismo que resulta al considerarlo con signo negativo. Para fines de diseño solo interesa el máximo valor de la respuesta bi-direccional, el cual ocurre cuando $\beta$ es positivo (figura $4 a$ ).

La máxima respuesta bi-direccional que resulta al considerar diferentes posibles orientaciones de los ejes en los que se descompone el movimiento del suelo (ejes de la estructura), se obtiene maximizando las ecs. 22 y 23 respecto a $k$, la cual aparece en el cálculo de $\beta$. (ec. 24). Se puede observar que la condición crítica ocurre para $k=1$. Este resultado es evidente tomando en consideración que la máxima correlación cruzada entre los componentes del movimiento del suelo corresponde a $k=1$ (Valdés, 2004).

Las figuras 4a y 4b muestran la variación de $\gamma$ en función de $\beta$, para diferentes valores de la parte real de la coherencia y $k=1$. En el caso donde los componentes de la respuesta son colineales (figura $4 a$ ), se observa lo siguiente: i) $\gamma$ no es simétrica respecto a $\beta=0$ y en todos los casos los mayores valores de $\gamma$ se obtienen para valores positivos de $\beta$. ii) Algunos valores de $\gamma$ son menores a uno. Esto significa que bajo ciertas combinaciones de signo para los componentes de la excitación, los efectos sísmicos bidireccionales resultan favorables. Sin embargo, si el objetivo es encontrar la máxima respuesta (fines de diseño), siempre existe una combinación de signos que garantiza un valor de $\gamma \geq 1$.0. iii) Los valores de $\gamma$ crecen en la medida que también crece el valor de la parte real de la coherencia. De igual forma, $\gamma$ crece $o$ decrece conforme $\beta$ tiende a $10-1$, respectivamente.

En el caso donde los componentes de la respuesta son ortogonales (figura 4b), se observa lo siguiente: i) $\gamma$ es simétrica respecto a $\beta=0$. ii) $\gamma$ crece conforme $\beta$ tiende a $10-1$. iii) $\gamma$ crece en la medida en que la parte real de la coherencia también crece. iv) $\gamma$ es siempre mayor a uno.

Comparando ambas gráficas (figuras 4a y 4b) se observa que los mayores valores de $\gamma$ se presentan cuando los componentes de la respuesta son colineales.

En los casos donde se prefiera utilizar una regla de combinación de porcentajes que considere el $100 \%$ del componente de la respuesta debido a la acción del sismo en una dirección $(x)$ y un porcentaje $\alpha$ del componente de la respuesta debido a la acción del sismo en la otra dirección (y) (ec. 4), entonces $\alpha$ puede calcularse de la siguiente forma.

Si los componentes de la respuesta son colineales 
$\alpha_{c}=\frac{ \pm \sqrt{1+\beta^{2}+2 \beta \operatorname{real}\left[\phi\left(\omega_{s}\right)\right]-1}}{\beta}$

Si los componentes de la respuesta son ortogonales

$\alpha_{o}= \pm \sqrt{\frac{ \pm \sqrt{1+\beta^{4}+2 \beta^{2} \text { real }^{2}\left[\phi\left(\omega_{s}\right)\right]-1}}{\beta^{2}}}$

\section{Ecuación 22}

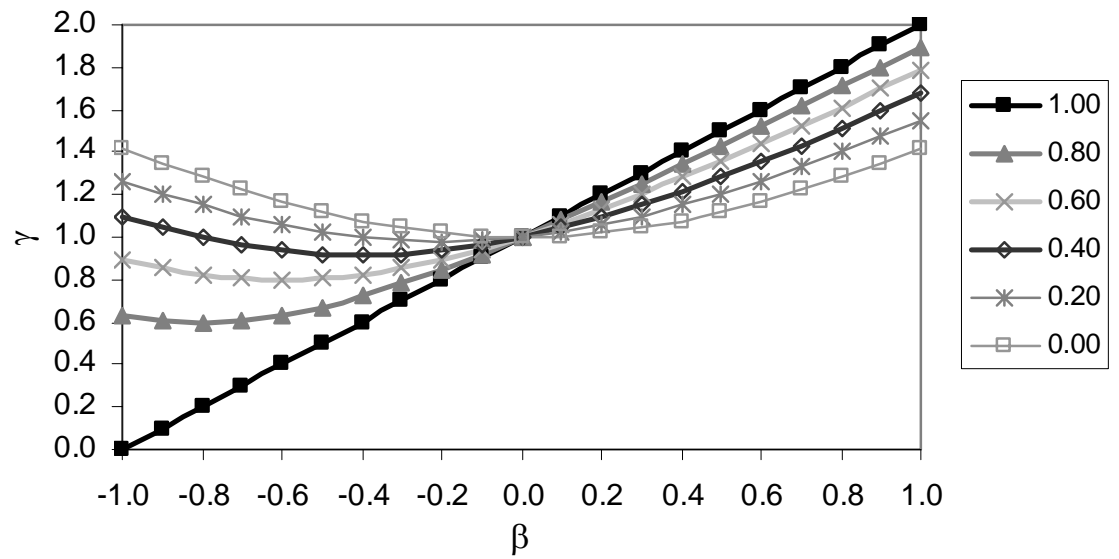

a) Componentes colineales de la respuesta

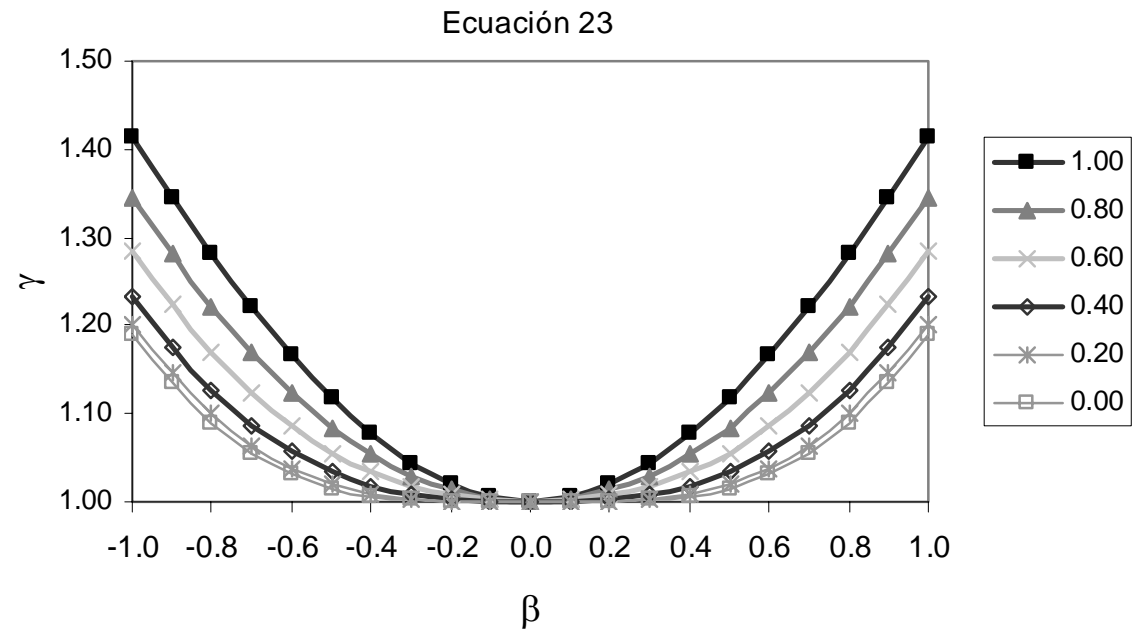

b) Componentes ortogonales de la respuesta

Figura 4. Variación de $\gamma$ en función de $\beta$. Los valores que se analizan de la parte real de la coherencia son: $0,0.2,0.4,0.6,0.8$ y 1.0. En todos los casos $k=1$ 
Estas expresiones (ecs. 25 y 26) se obtuvieron bajo las mismas consideraciones y en forma similar a las ecs. 22 y 23. En este caso, se substituyeron las ecs. 14, 15, 18 y 19 en la ec. 4.

Las figuras 5a y 5b muestran la variación de $\alpha$ en función de $\beta$ para diferentes valores de la parte real de la coherencia $\varphi(\omega)$ y $k=1$. Se observa que $\alpha$ crece conforme $\beta$ tiende a 1 en el caso de componentes colineales (figura 5a) y a 1 o -1 para componentes ortogonales (figura 5b). También se observa un incremento de $\alpha$ cuando la parte real de la coherencia aumenta.

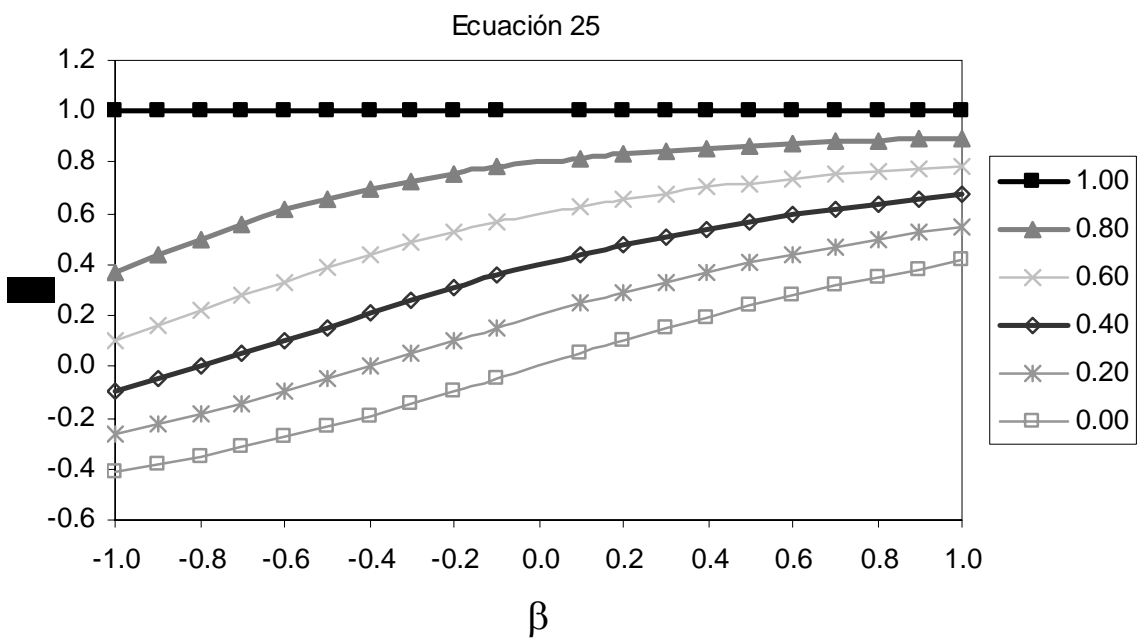

a) Componentes colineales de la respuesta

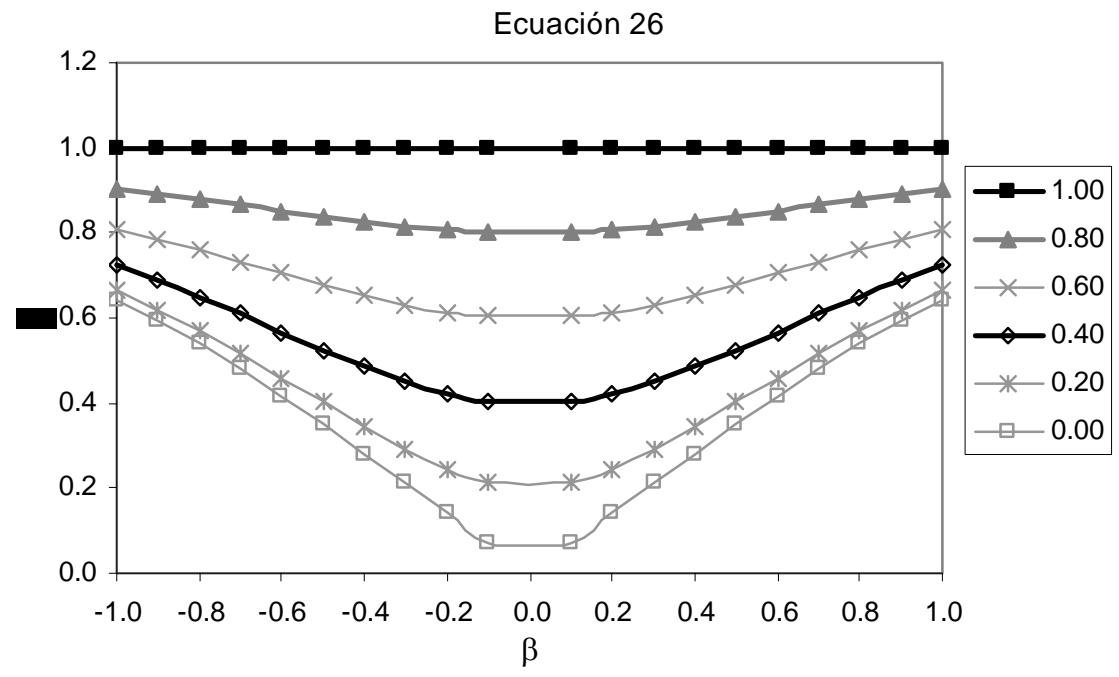

b) Componentes ortogonales de la respuesta

Figura 5. Variación de $\alpha$ en función de $\beta$. Los valores que se analizan de la parte real de la coherencia son: $0,0.2,0.4,0.6,0.8$ y 1.0. En todos los casos $k=1$ 
Para el caso de componentes colineales, $\alpha$ varía de 0 a 1 . Para el caso de componentes ortogonales, $\alpha$ varía de 0.64 a 1 . Los mayores valores de $\alpha$ se presentan cuando los componentes de la respuesta son colineales.

\section{CÁLCULO DE LAS FUNCIONES $g_{1}(\omega)$ y $g_{2}(\omega)$}

De acuerdo con las ecs. 18 y 19, el cociente de las máximas respuestas considerando la acción unidireccional del movimiento del suelo es el siguiente:

$$
\frac{E\left(R_{x \max }\right)}{E\left(R_{y \text { max }}\right)}=\frac{F_{x}}{F_{y}}\left(\frac{\sqrt{E\left(R_{x \max }^{2}\right)}}{\left.\sqrt{E\left(R_{y \text { max }}^{2}\right)}\right)}\right)=\frac{F_{x}}{F_{y}} \sqrt{\frac{\frac{F_{p x} g_{1}^{2}\left(\omega_{s}\right)}{2 \pi T_{s x}}}{\frac{F_{p y} k^{2} g_{2}^{2}\left(\omega_{s}\right)}{2 \pi T_{s y}}}}
$$

donde $F_{x}$ y $F_{y}$ son funciones que transforman la raíz cuadrada del valor esperado de la máxima respuesta cuadrática en el valor esperado de la máxima respuesta. Si se considera que los factores pico y la duración de la fase intensa del movimiento del suelo son los mismos en el numerador y en el denominador, además de que $F_{x} / F_{y}=1$, entonces

$$
\frac{E\left(R_{x \max }\right)}{E\left(R_{y \max }\right)}=\frac{g_{1}\left(\omega_{s}\right)}{g_{2}\left(\omega_{s}\right) k}=\beta
$$

De esta forma, $\beta$ es el cociente de las respuestas unidireccionales pico debidas a la acción individual del sismo en la dirección $x$ o $y$. Esta conclusión es importante, ya que las respuestas individuales pico ( $x$ o y) pueden calcularse por medio de las técnicas usuales de espectros de respuesta..

\section{EJEMPLOS DE APLICACIÓN}

Se resuelven dos ejemplos para verificar la precisión del procedimiento expuesto en este trabajo. En el primer ejemplo se analiza un modelo de tres grados de libertad (dos desplazamientos ortogonales horizontales y un giro). En el segundo ejemplo se analiza un edificio de concreto reforzado de cuatro niveles. El principal objetivo de estos ejemplos es comparar los resultados obtenidos utilizando el procedimiento expuesto en este trabajo y las reglas de combinación existentes, respecto a la respuesta bidireccional "exacta", la cual corresponde a un análisis elástico, bi-direccional, paso a paso. Los acelerogramas que se utilizan corresponden a distintas estaciones de terreno blando de la ciudad de México.

\section{Ejemplo 1}

La figura 6 muestra el modelo de tres grados de libertad que se analiza en este ejemplo. Las variables que definen el modelo son: $a, m, K_{x}, B_{x}=e_{x} / a, B_{y}=\mathrm{e}_{y} / a r, r=b / a$ y $\lambda=K_{y} / K_{x}$. Donde $K_{x}$ y $K_{y}$ son las rigideces de traslación en las direcciones ortogonales, $e_{x}$ y $e_{y}$ la excentricidad en cada dirección, $a$ y $b$ las dimensiones en planta del modelo y $m$ la masa traslacional. 
Se analizan tres juegos de parámetros representativos de edificios convencionales: Modelo $\mathrm{A}(\lambda=r$ $\left.=1 / 2, B_{x}=0.2\right)$, Modelo B $\left(\lambda=r=1 / 5, B_{x}=0.35\right)$ y Modelo C $\left(\lambda=r=1, B_{x}=0\right)$. Para los tres modelos se asume $m=1.00 \mathrm{Ton} / \mathrm{m} / \mathrm{s}^{2}, a=1.00 \mathrm{~m}, K_{x}=9.8696 \mathrm{Ton} / \mathrm{m}\left(T_{x}=2.00 \mathrm{~s}\right.$ ), $\xi=0.05$ (relación de amortiguamiento crítico), $k=1$ y $0 \leq B_{y} \leq 0.5$. La respuesta estructural que se analiza corresponde a la fuerza cortante que actúa en alguno de los marcos del modelo. Para el modelo A se analiza el marco 4, para el modelo B el marco 1 y para el modelo C el marco 2 (figura 6).

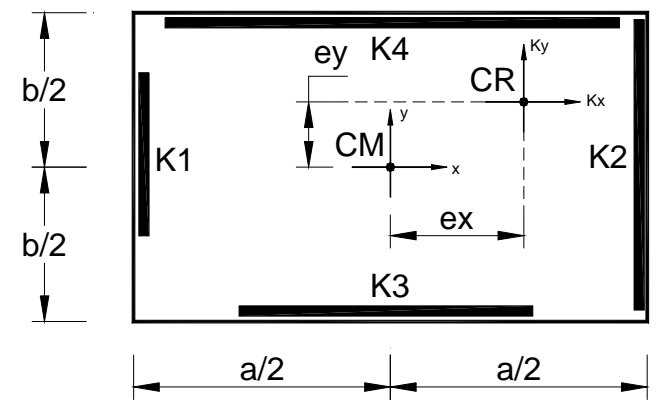

Figura 6. Vista en planta del modelo que se analiza en el ejemplo 1

Primero, se muestra la aplicación del procedimiento propuesto en este artículo mediante el análisis de un caso especial para el modelo $B$, en el cual $B_{y}=0.0527$. Como resultado del análisis modal unidireccional para el acelerograma del temblor del 19 de septiembre de $1985\left(\mathrm{M}_{\mathrm{s}}=8.1\right)$, obtenido en suelo blando de la ciudad de México (estación SCT, $T_{s}=2.0 \mathrm{~s}$ ), se obtienen los siguientes resultados correspondientes a la fuerza cortante que actúa sobre el marco 1: $V_{1}= \pm 0.604$ Ton (sismo en la dirección $x$ ) y $V_{1}= \pm 0.602$ Ton (sismo en la dirección y). Por otra parte, los resultados obtenidos del análisis elástico, bi-direccional, paso a paso, son los siguientes: $V_{1}=1.02$ Ton (sismo actuando en la direcciones $x$ y $y$, simultáneamente), $V_{1}=0.757$ Ton (sismo actuando en $x$ y $-y$ ), $V_{1}=-0.757$ Ton (sismo en $-x$ y $y$ ) y $\mathrm{V}_{1}=$ 1.02 Ton (sismo en $-x$ y $-y$ ).

El objetivo es combinar los resultados del análisis modal unidireccional para obtener una estimación de los resultados obtenidos en el análisis elástico, bi-direccional, paso a paso. Para fines de diseño el resultado bi-direccional que interesa es $V_{1}= \pm 1.02$ Ton.

De acuerdo al método SRSS, la máxima fuerza cortante bi-direccional que se obtiene es $V_{\text {MAX,SRSS }}=\sqrt{(0.604)^{2}+(0.602)^{2}}=0.8527$ Ton . Si se utiliza una regla de combinación para $\alpha=0.30$ (RDF 1993), entonces $V_{M A X, \alpha=0.30}=0.604+(0.3)(0.602)=0.7846$ Ton..

La regla CQC3 es otra regla que puede utilizarse para calcular los efectos sísmicos bi-direccionales (Menun y Der Kiureghian, 1998; Smeby y Der Kiureghian, 1985). Esta regla toma en consideración, explícitamente, la correlación entre las respuestas modales, además de la correlación entre los componentes del movimiento del suelo. Esta regla permite calcular la respuesta bi-direccional máxima, como función del ángulo de incidencia del temblor. Para simplificar su aplicación, se ha propuesto una fórmula simplificada que permite calcular en forma directa la máxima respuesta bi-direccional $\left(r_{c r}\right)$, sin necesidad de calcular explícitamente el ángulo crítico de incidencia del temblor (López y Torres, 1997; López et al., 2000). Para fines de este artículo, la regla CQC3 se define mediante la expresión simplificada que se comenta. 


$$
r_{c r}=r_{x}\left(\left(1+k^{2}\right)\left(\frac{1+\beta_{C Q C}^{2}}{2}\right)+\left(1-k^{2}\right)\left(\frac{1-\beta_{C Q C}^{2}}{2}\right)^{2}+\left(\frac{r_{x y}}{r_{x} r_{y}}\right)^{2} \beta_{C Q C}^{2}\right)^{\frac{1}{2}}
$$

donde $k$ es el cociente del espectro de respuesta que se utilice para el análisis en la dirección $x$, dividido entre el espectro de respuesta para la dirección $y, \beta_{\mathrm{CQC}}=r_{y} / r_{\mathrm{x}}, r_{x}$ y $r_{y}$ son las respuestas unidireccionales en las direcciones indicadas y $r_{x y}$ la covarianza entre las respuestas unidireccionales $r_{x}$ y $r_{y}$, la cual se calcula de la siguiente forma

$$
r_{x y}=\sum_{i} \sum_{j} \rho_{i j} r_{x i} r_{y j}
$$

donde

$$
r_{k}=\left(\sum_{i} \sum_{j} \rho_{i j} r_{k i} r_{k j}\right)^{\frac{1}{2}} ; \quad k=x, y
$$

$r_{i j}$ es el coeficiente de correlación modal entre los modos $i$ y $j$.

Analizando el intervalo que contiene a los distintos valores de la respuesta crítica $\left(r_{c r}\right)$, que se pueden calcular con la ec. 29, para todos los posibles valores de $\beta_{C Q C}, k$ y $\mathrm{r}_{\mathrm{xy}} / \mathrm{r}_{\mathrm{x}} \mathrm{r}_{\mathrm{y}}$, se observa que: $r_{x} \leq r_{c r} \leq \sqrt{2} r_{x}$, lo cual en términos de $\gamma$ representa una variación de $1 \leq \gamma \leq \sqrt{2}$. Los máximos valores para la respuesta crítica bi-direccional que se pueden calcular con la ec. 29, son los mismos que se pueden calcular mediante la regla SRSS. La regla CQC3 (ec. 29) nunca dará mayores valores para la respuesta crítica bi-direccional que los que se obtengan con la regla SRSS. Para este caso particular, la mayor fuerza cortante que puede calcularse aplicando la ec. 29, se obtiene para los siguientes valores de las variables involucradas en el cálculo, $k=1, \beta_{C Q C}=0.602 / 0.604=0.996$ y $\mathrm{r}_{\mathrm{xy}} / \mathrm{r}_{\mathrm{x}} \mathrm{r}_{\mathrm{y}}=1$, los cuales hacen que: $\mathrm{V}_{\mathrm{CQCB}}=\left(\sqrt{1+(0.9966)^{2}}\right)(0.604)=0.8527$ Ton.

La regla SRSS arroja un error al comparar la estimación de la máxima respuesta bi-direccional calculada con esta regla, respecto a la máxima respuesta bi-direccional calculada mediante el análisis elástico, bi-direccional, paso a paso, de $19.6 \%$. La regla de combinación de porcentajes para $\alpha=0.3$ arroja un error de 30\%. El error de la estimación hecha con la ec. 29 es el mismo que el de la regla SRSS. Todas las reglas subestiman la respuesta bi-direccional crítica.

Si se utiliza el procedimiento que se presenta en este artículo, el cálculo de la máxima respuesta bidireccional se lleva a cabo de la siguiente forma. Primero se calcula el cociente $\beta$ (ес. 24) a partir de las máximas respuestas unidireccionales $\beta_{1}=0.602 / 0.604=0.9966$ y $\beta_{2}=-0.9966$. Como en este caso los componentes de la respuesta son colineales, entonces se utiliza la ec. 22, la cual para un valor de la parte real de la coherencia de 0.4 proporciona un valor de $\gamma_{1}=1.6704$ y $\gamma_{2}=1.09$. La máxima fuerza cortante 
para el marco 1 del modelo B, considerando la acción simultánea de ambos componentes ortogonales, horizontales del movimiento del suelo, se calcula multiplicando a $\gamma$ por la mayor de las dos respuestas unidireccionales. De donde resulta: $V_{\gamma 1}=1.6704(0.604)=1.009$ Ton y $V_{\gamma 2}=1.09(0.604)=0.658$ Ton. En este caso, para $V_{\gamma 1}$, el error de la estimación es de $1 \%$. $V_{\gamma 2}$ corresponde a la máxima respuesta bidireccional para la otra posible combinación de signos de los componentes del movimiento del suelo y no tiene importancia para fines de diseño.

A continuación se muestran los resultados paramétricos de los casos analizados. La figura 7 muestra los resultados para los tres modelos analizados en el caso 1, en el cual se utilizan los registros sísmicos del 19 de septiembre de $1985\left(\mathrm{M}_{\mathrm{s}}=8.1\right)$ registrados en la estación SCT $\left(\mathrm{T}_{\mathrm{s}}=2.0 \mathrm{~s}\right)$. Los resultados se presentan en términos del error de las estimaciones hechas con las distintas reglas consideradas, incluyendo la desarrollada en este trabajo; respecto a los resultados de los análisis, elásticos, bi-direccionales, paso a paso ("respuesta verdadera”).

Para el modelo A (figura 7a), se observa que el error que resulta al aplicar el procedimiento $\gamma$ es menor al 5\% para todos los valores de $B_{y}$, mientras que los errores de las reglas SRSS y $\alpha=0.3$ pueden llegar a ser de hasta $15 \%$ y $10 \%$, respectivamente. Un signo negativo del error, significa que la máxima respuesta bi-direccional se subestima.

Para el modelo B (figura 7b), se observa que el error para las reglas SRSS y $\alpha=0.3$ son mayores a los que se obtienen para el modelo A (figura 7a). El mayor error para la regla SRSS es de alrededor del $20 \%$ y para la regla $\alpha=0.3$ de 30\%. Los errores que resultan en este modelo al utilizar el procedimiento $\gamma$ son similares a los del modelo A.

La figura 7c muestra los resultados para el modelo C. En este modelo del error que resulta al utilizar el procedimiento $\gamma$ es prácticamente de cero. Las otras reglas alcanzan errores de hasta $20 \%$.

En la figura 8a se presentan los resultados para el modelo C correspondientes al caso de análisis 2. En este caso se analizan los registros sísmicos del mismo temblor que en el caso anterior pero registrados en distinto sitio (Central de abastos, $\mathrm{T}_{\mathrm{s}}=4.0 \mathrm{~s}$ ). Los parámetros estructurales del modelo son los mismos, solo se modifica el periodo desacoplado de traslación en la dirección $x$, siendo en este caso $T_{x}=4.0 \mathrm{~s}$

En la figura 8b se muestran los resultados del caso 3 (estación 11, $\mathrm{T}_{\mathrm{s}}=4.3 \mathrm{~s}$ ). Los acelerogramas que se analizan en este caso corresponden al temblor del 25 de abril de $1989\left(\mathrm{M}_{\mathrm{s}}=6.9\right)$. El modelo y sus propiedades estructurales son las mismas que en el caso 2.

Para estos dos casos de análisis (2 y 3) el error en la estimación de la máxima respuesta bidireccional estimada mediante el procedimiento g resulta menor al 5\%.

Se observa para este ejemplo, que el procedimiento $\gamma$ proporciona menores errores en la estimación de la máxima respuesta bi-direccional, en comparación a las reglas existentes. Para estos cálculos se ha considerado que la parte real de la coherencia entre los componentes del movimiento del suelo es de 0.6 (Valdés, 1999 y 2003; Santa-Cruz et al., 1999). 


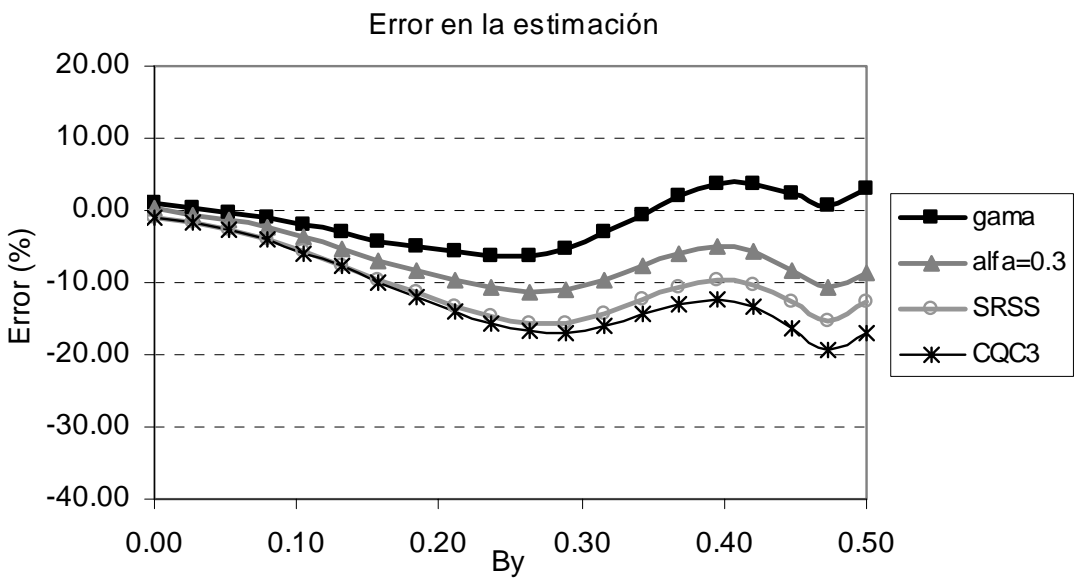

a) Modelo A, marco 4

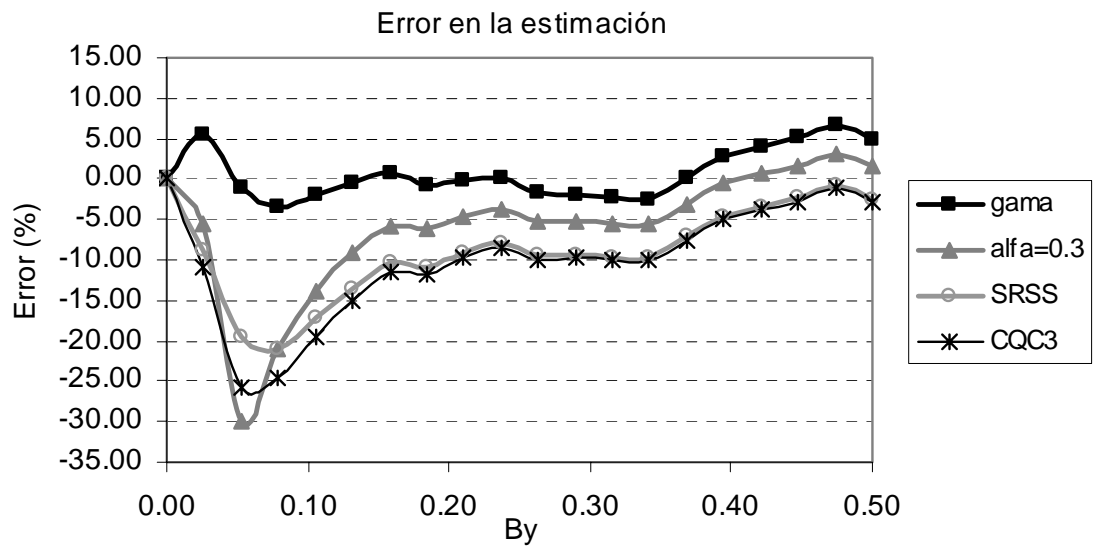

b) Modelo B, marco 1

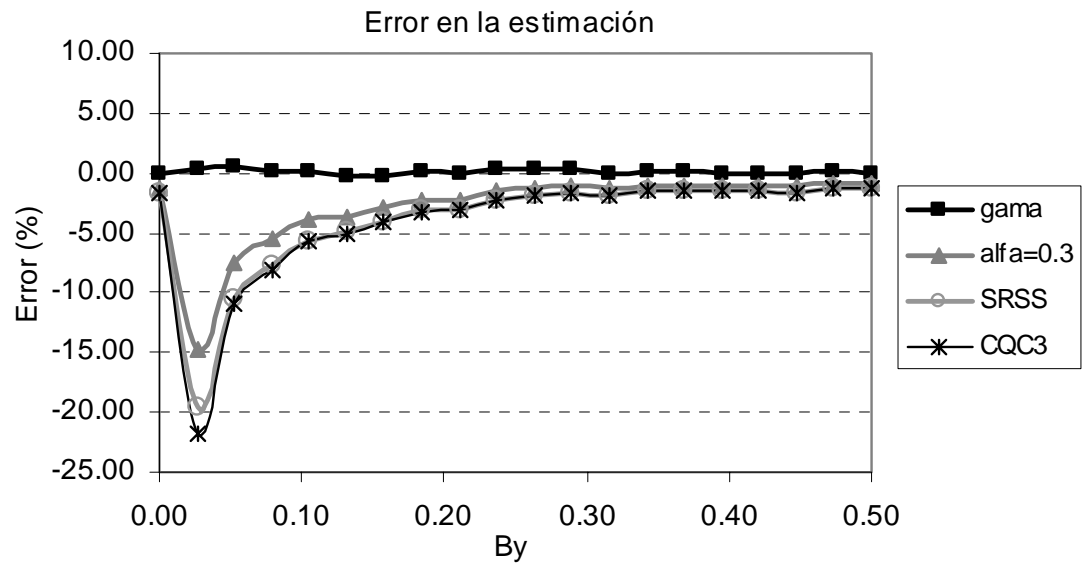

c) Modelo C, marco 2

Figura 7. Errores en la estimación de la máxima fuerza cortante bi-direccional que actúa en algún marco estructural de los modelos analizados en el ejemplo 1, para diferentes reglas de combinación 


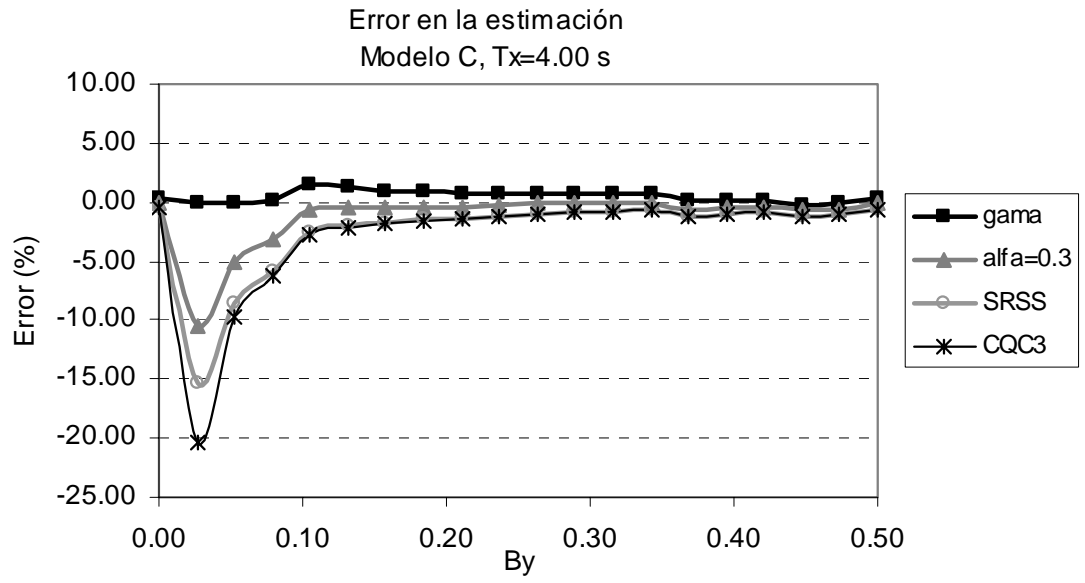

a) Caso 2 (Central de Abastos, $\left.T_{\mathrm{s}}=4.00 \mathrm{~s}\right)$

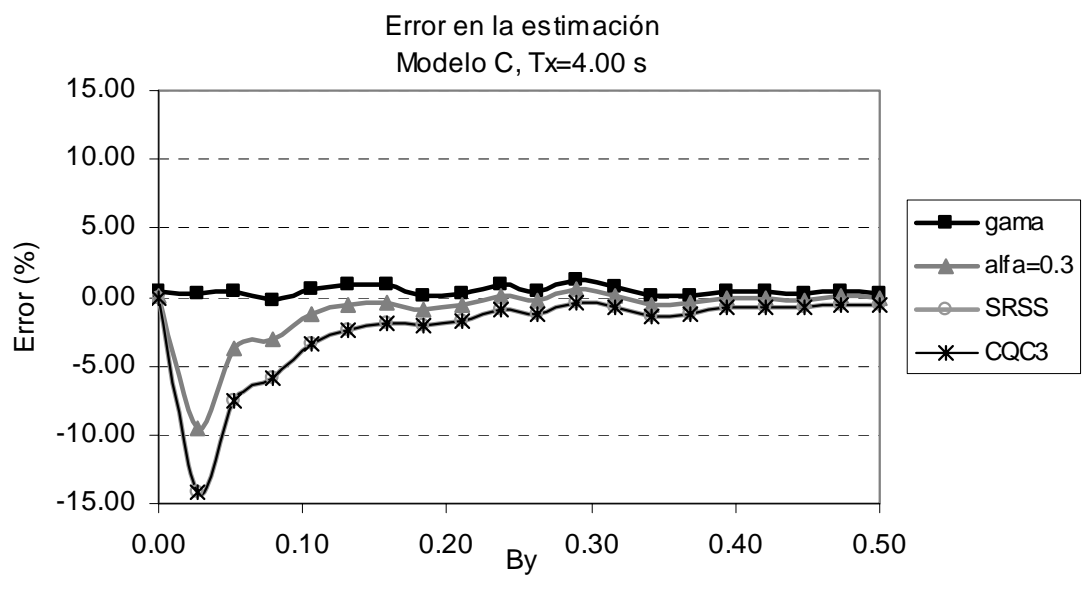

b) Caso 3 (estación 11, $\mathrm{T}_{\mathrm{s}}=4.30 \mathrm{~s}$ )

Figura 8. Errores en la estimación de la máxima fuerza cortante bi-direccional que actúa en el marco 2 del modelo C, utilizando acelerogramas registrados en diferentes sitios de terreno blando

\section{Ejemplo 2}

En la figura 9 se muestra la estructura que se analiza en este ejemplo, la cual corresponde a un edificio de concreto reforzado de cuatro niveles. Se analizan dos respuestas, el esfuerzo normal en la columna de esquina identificada como columna A, y el cortante basal. Se utilizan los acelerogramas registrados en la estación SCT de la ciudad de México $\left(\mathrm{T}_{\mathrm{s}}=2.0 \mathrm{~s}\right)$ registrados durante el temblor del 19 de septiembre de $1985\left(\mathrm{M}_{\mathrm{s}}=8.1\right)$, los cuales se rotaron para analizar la dirección de máxima correlación cruzada entre los componentes del movimiento del suelo $(k=1)$. Se analizaron todas las posibles combinaciones de signo para la excitación y se consideró una parte real de la coherencia de 0.6. 
El esfuerzo normal en la columna A es ocasionado por la acción de la fuerza axial y por el momento de flexión bi-direccional. En este caso el tipo de respuesta que se analiza es colineal. En el caso de la fuerza cortante basal, el tipo de respuesta es ortogonal.

Los resultados de este ejemplo muestran que el error en la estimación del esfuerzo normal en la columna A, al utilizar la regla $100 \%+30 \%$ es de $-32.55 \%$, mientras que con la regla SRSS, el error es de $27.51 \%$. Por su parte, el error que resulta al utilizar el procedimiento $\gamma$ es de $-8.23 \%$.

Para otras respuestas cuya estimación individual no tiene utilidad práctica (carga axial y momentos flexionantes), pero que permiten comparar las diferentes reglas de combinación, se observa que los errores en la estimación de la carga axial bi-direccional son similares a los correspondientes al esfuerzo normal, mientras que para el caso del momento flexionante alrededor del eje $x$ de la columna $\left(M_{x}\right)$, los errores que resultan al aplicar los tres procedimientos son del mismo orden (menores a $-2.3 \%$ ). Para $M_{y}$, el mayor error resulta al aplicar la regla SRSS (-15.70\%) mientras que el menor al utilizar el procedimiento $\gamma(-$ $4.33 \%)$.

En todos los casos, el menor error en la estimación de la máxima respuesta bi-direccional se obtiene al utilizar el procedimiento $\gamma$.

Respecto a la fuerza cortante basal bi-direccional, los errores en las estimaciones que se obtuvieron al aplicar las distintas reglas fueron de $-4.72 \%$ para el procedimiento $\gamma$ y de $-18.51 \%$ para la regla $100 \%+30 \%$. La regla SRSS arrojó un error similar al del procedimiento $\gamma$, solo que con signo positivo, lo cual indica que la respuesta se sobreestima. En caso de utilizar la regla CQC3 y seleccionar apropiadamente los parámetros involucrados en esta fórmula, se puede llegar a alcanzar un error de hasta $0 \%$ en la estimación del cortante basal bi-direccional.

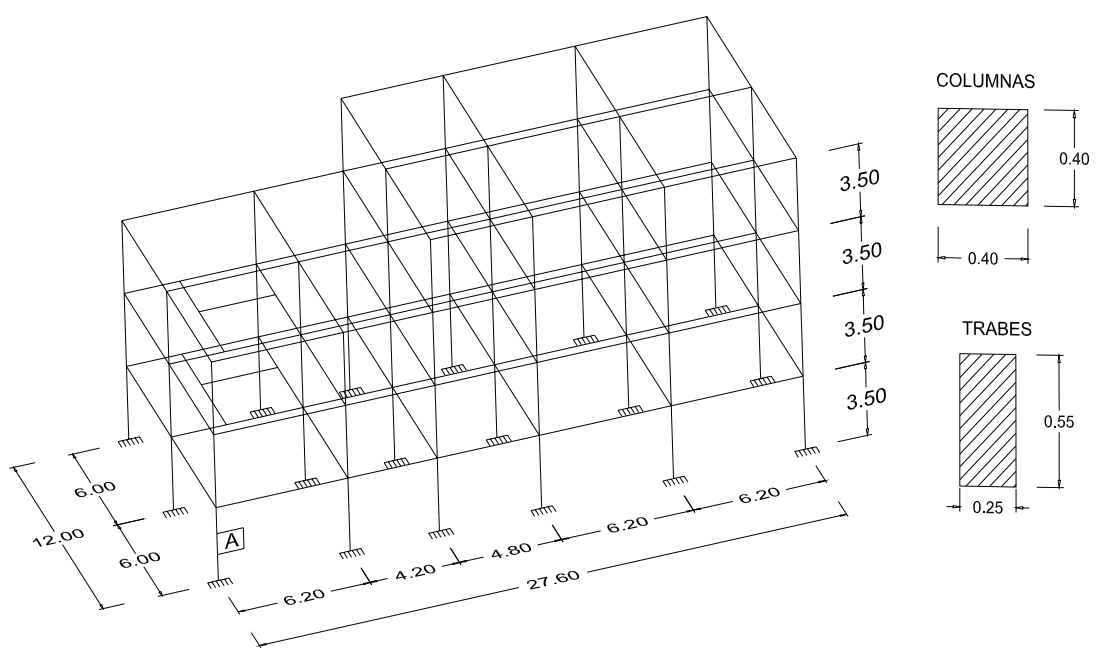

Figura 9. Modelo del edificio que se analiza en el ejemplo 2

\section{CONCLUSIONES}

Se desarrolló un procedimiento para estimar la máxima respuesta bi-direccional de las estructuras desplantadas en terreno blando. Las estimaciones hechas con este procedimiento se compararon respecto a 
los resultados de un análisis elástico, bi-direccional, paso a paso para diferentes casos de estudio. El procedimiento toma en cuenta el tipo de respuesta de que se trate (colineal u ortogonal), así como la orientación de los ejes ortogonales de la estructura a lo largo de los cuales se descompone el movimiento del terreno. La principal hipótesis que se hizo en la formulación del procedimiento expuesto, fue utilizar deltas de Dirac para representar los espectros de amplitudes de Fourier correspondientes a los dos componentes ortogonales horizontales del movimiento del suelo.

Las expresiones desarrolladas que permiten estimar la máxima respuesta bi-direccional de la estructura que se analiza (ecs. 22 a 26), son función de dos variables: $\beta$ la cual representa al cociente de las respuestas máximas individuales para la acción unidireccional del movimiento del suelo, y $\varphi$ que representa la parte real de la coherencia suavizada entre los componentes ortogonales horizontales del movimiento del suelo. La orientación de los ejes de la estructura se toma en cuenta por medio de la variable $k$, la cual se define como el cociente de las amplitudes de Fourier correspondientes a ambos componentes del movimiento del suelo. $k$ afecta el cálculo de $\beta$ (ec. 24).

En general, se observa que la respuesta sísmica bi-direccional depende de las características dinámicas de la estructura, de las características del movimiento del suelo y del tipo de respuesta que se analice (ortogonal o colineal). Ninguno de los procedimientos existentes toma en cuenta explícitamente, ni las características del movimiento del suelo (duro o blando), ni el tipo de respuesta. Para los dos ejemplos analizados en este artículo, las fuerzas de diseño estimadas mediante las reglas existentes resultaron sistemáticamente menores a las fuerzas que se obtuvieron de los análisis elásticos, bi-direccionales, paso a paso realizados. Por su parte, el método propuesto, el cual involucra el cálculo de $\gamma$ ( $\alpha$ alternativamente), proporciona errores menores en las estimaciones hechas para las diferentes respuestas analizadas, en comparación a los procedimientos existentes (tabla 1). Es importante tomar en cuenta que el procedimiento propuesto, es aplicable en forma exclusiva al caso de terreno blando.

Tomando en cuenta los dos ejemplos analizados en este trabajo, se observa que las reglas existentes consideran solo algunos casos que pueden presentarse al tratar de combinar los efectos sísmicos ortogonales. En general, la regla SRSS sobreestima la respuesta máxima bi-direccional cuando los componentes de la respuesta son ortogonales y la subestima cuando son colineales. La mayor respuesta bidireccional que se puede calcular utilizando la regla CQC3 es la misma que se obtiene de la regla SRSS. Por lo tanto, cuando se combinan componentes ortogonales de la respuesta, la regla CQC3 puede ser exacta en el cálculo de la máxima respuesta bi-direccional, pero en el caso de componentes colineales, la regla CQC3 puede subestimar la respuesta (Ejemplo 1).

Las reglas de combinación de porcentajes $(100 \%+30 \%$ y 100\%+40\%) representan algunos casos para valores particulares de $\gamma \mathrm{y} \varphi$, dependiendo del tipo de respuesta que se analice. Todos los casos y tipos de respuesta pueden representarse en forma razonable y práctica, mediante el procedimiento propuesto en este artículo.

Tabla 1. Errores en las estimaciones de las máximas respuestas elásticas, bi-direccionales

\begin{tabular}{cccc}
\hline Regla propuesta & Regla 100\%+30\% & $\begin{array}{c}\text { Regla } \\
\text { SRSS }\end{array}$ & Modelo y respuesta estructural que se analiza \\
\hline$-8.30 \%$ & $-32.50 \%$ & $-27.50 \%$ & $\begin{array}{c}\text { Esfuerzo normal en una columna de esquina de un edificio de } \\
\text { cuatro niveles (ejemplo 2). }\end{array}$ \\
$-5.00 \%$ & $-18.50 \%$ & $5.00 \%$ & $\begin{array}{c}\text { Fuerza cortante basal en un edificio de cuatro niveles } \\
\text { (ejemplo 2). }\end{array}$ \\
$-1.00 \%$ & $-30.00 \%$ & $-20.00 \%$ & $\begin{array}{c}\text { Fuerza cortante en un marco estructural de un modelo de tres } \\
\text { grados de libertad (ejemplo 1, caso 1) }\end{array}$ \\
\hline
\end{tabular}




\section{REFERENCIAS}

American Society of Civil Engineers (ASCE), 1986. "Seismic Analysis of Safety Nuclear Structures and Commentary on Standard for Seismic Analysis of Safety Related Nuclear Structures”, New York.

Building Seismic Safety Council. NEHRP, 1997. "Recommended provisions for seismic regulations for new buildings”. FEMA302, Washington, DC.

California Department of Transportation (Caltrans), 1990. “Bridge Design Specifications”, Sacramento, CA.

COVENIN, 1998. Edificaciones Sismorresistentes. “Norma Venezolana COVENIN 1756”.

Heredia-Zavoni, E y R Machicao-Barrionuevo (2004), "Response to orthogonal components of ground motion and assessment of percentage combination rules". Earthquake Engineering and Structural Dynamics, Vol. 33, pp. 271-284.

International Conference of Building Officials (ICBO), 1997. "Uniform Building Code”, Vol. 2, Structural Engineering Design Provisions, Whittier, CA., 492 pp

López, O A y R Torres (1997), "The critical angle of seismic incidence and the maximum structural response”. Earthquake Engineering and Structural Dynamics, Vol. 26, pp. 881-894.

López, O A, A K Chopra, y J J Hernández (2000), “Critical response of structures to multicomponent earthquake excitation”. Earthquake Engineering and Structural Dynamics, Vol. 29(12), pp. 17591778.

Menun, C y A Der Kiureghian (1998), "A replacement for the 30\%, 40\% and SRSS rules for multicomponent seismic analysis”. Earthquake Spectra, Vol. 14, No. 1, pp. 153-156.

Newmark, N M (1975), "Seismic design criteria for structures and facilities, trans-Alaska pipeline system”. Proceedings of the U.S. National Conference Earthquake Engineering, EERI, pp. 94-103.

Nutt, R V (1996), “ATC-32: Provisional recommendations for the seismic design of bridges". Proceedings of the 4th Caltrans Seismic Workshop, California Department of Transportation, Sacramento, CA.

Reed, J W y R P Kennedy (1996), "Discussion of "A Clarification of Orthogonal Effects in a TreeDimensional Seismic Analysis” by E.L. Wilson, I. Suharwardy, and A. Habibullah”. Earthquake Spectra Vol. 12, No. 2, pp. 353-356.

“Reglamento de Construcciones para el Distrito Federal (RDF)", 1993. Departamento del Distrito Federal, México.

Reyes-Salazar, A, J A Juárez-Duarte, A López-Barraza, y J I Velázquez-Dimas (2004), “Combined effect of the horizontal components of earthquakes for moment resisting steel frames". Steel and Composite Structure An International Journal, Vol. 4, No 3, pp. 189-209.

Rosenblueth, E y H Contreras (1977). “Approximate design for multicomponent earthquakes”. Journal of the Engineering Mechanics, Division (ASCE), Vol. 103, pp. 881-893.

Santa Cruz, S, E Heredia-Zavoni, y R S Harichandran (1999), "Variación de la coherencia del movimiento sísmico del terreno en frecuencias bajas y modelos para el Valle de México”, Memorias del XII Congreso Nacional de Ingeniería Sísmica, Vol. 1, Morelia- Michoacán, México, pp. 197-206. 
Santa Cruz, S C (1999), "Simulación condicional de campos aleatorios: aplicaciones al movimiento sísmico del terreno”, Tesis de Maestría, Universidad Nacional Autónoma de México.

Smeby, W y A Der Kiureghian (1985), "Modal combination rules for multicomponent earthquake excitation”. Earthquake Engineering and Structural Dynamics, Vol. 13, pp.1-12

Valdés, J (1999), “Análisis de efectos sísmicos ortogonales horizontales”. Tesis de Maestría, Posgrado en Ingeniería, UNAM, México.

Valdés, J (2004), “Análisis de efectos sísmicos ortogonales horizontales en terreno blando de la ciudad de México”. Tesis de Doctorado, Posgrado en Ingeniería, UNAM, México.

Valdés, J (2003), “Análisis de la coherencia entre los componentes ortogonales horizontales del movimiento sísmico del terreno en el valle de México y en la costa del Pacífico mexicano", Memorias del XIV Congreso Nacional de Ingeniería Sísmica, León-Guanajuato, México, pp. 27-39.

Vanmarcke, E H (1976), Seismic risk and engineering decisions (Structural response to earthquakes), C. Lomnitz and E. Rosenblueth, Editors. Elsevier Publishing Co., pp. 287-337. Amsterdam.

Wilson, E L, I Suharwardy, y A Habibulla (1995), "A clarification of the orthogonal effects in a threedimensional seismic analysis”. Earthquake Spectra, Vol. 11, No. 4, pp. 659-666 